\title{
Tandem Oxidation of Allylic and Benzylic Alcohols to Esters Catalyzed by $N$-Heterocyclic Carbenes
}

\author{
Brooks E. Maki, Audrey Chan, Eric M. Phillips, Karl A. Scheidt* \\ Department of Chemistry, Northwestern University, \\ 2145 Sheridan Road, Evanston, Illinois, 60208

\section{Supporting Information}

1. General methods

2. Procedure and characterization data for reactions with alcohol as solvent

3. Procedure and characterization data for reactions with toluene as solvent

4. Procedure and characterization data for asymmetric reactions and catalyst

5. Selected NMR Spectra
$\mathrm{S} 1-\mathrm{S} 2$

S2-S4

S4-S5

S5-S7

S8-S17

General Information. All reactions were carried out under a nitrogen atmosphere in flamedried glassware with magnetic stirring. Toluene, $\mathrm{MeOH}$, and $\mathrm{CH}_{2} \mathrm{Cl}_{2}$ were purified by passage through a bed of activated alumina. ${ }^{1}$ Reagents were purified prior to use unless otherwise stated following the guidelines of Perrin and Armarego. ${ }^{2}$ Purification of reaction products was carried out by flash chromatography using EM Reagent silica gel 60 (230-400 mesh). Analytical thin layer chromatography was performed on EM Reagent $0.25 \mathrm{~mm}$ silica gel 60-F plates. Visualization was accomplished with UV light and anisaldehyde, ceric ammonium nitrate stain, potassium permangenate, or phosphomolybdic acid followed by heating. Infrared spectra were recorded on a Perkin Elmer 1600 series FT-IR spectrometer. ${ }^{1} \mathrm{H}-\mathrm{NMR}$ spectra were recorded on a Varian Inova $500(500 \mathrm{MHz})$ or Mercury $400(400 \mathrm{MHz})$ spectrometer and are reported in ppm using solvent as an internal standard $\left(\mathrm{CDCl}_{3}\right.$ at $\left.7.26 \mathrm{ppm}\right)$. Data are reported as (ap = apparent, $\mathrm{s}$ $=$ singlet, $\mathrm{d}=$ doublet, $\mathrm{t}=$ triplet, $\mathrm{q}=$ quartet, $\mathrm{m}=$ multiplet, $\mathrm{b}=$ broad; coupling constant $(\mathrm{s})$ in $\mathrm{Hz}$; integration. Proton-decoupled ${ }^{13} \mathrm{C}-\mathrm{NMR}$ spectra were recorded on a Varian Inova 500 (125 $\mathrm{MHz})$ or Mercury $400(100 \mathrm{MHz})$ spectrometer and are reported in ppm using solvent as an

1. Pangborn, A. B.; Giardello, M. A.; Grubbs, R. H.; Rosen, R. K.; Timmers, F. J. Organometal. 1996, 15, 15181520.

2. Perrin, D. D. and Armarego, W. L. Purification of Laboratory Chemicals; 3rd Ed., Pergamon Press, Oxford. 1988. 
internal standard $\left(\mathrm{CDCl}_{3}\right.$ at $\left.77.0 \mathrm{ppm}\right)$. Mass spectra data were obtained on a Varian 1200 Quadrupole Mass Spectrometer and Micromass Quadro II Spectrometer.

3-Phenyl-butyn-1-ol was purchased from GFS Chemicals. Manganese(IV) oxide and all other alcohols were commercially available from Sigma-Aldrich Chemical Company. Triazolium salt 21 was prepared using the method of Rovis, et al. ${ }^{3}$ with mesitylhydrazine prepared using the method of Carlin and Moores. ${ }^{4}$

General Procedure for triazolium-catalyzed oxidation with alcohol as solvent:

A flame-dried round bottom flask was charged with triazolium salt $(22 \mathrm{mg}, 0.10 \mathrm{mmol})$. The flask was sealed with a septum and put under positive pressure of nitrogen. The allylic alcohol (1 $\mathrm{mmol})$, and the nucleophilic alcohol $(5 \mathrm{~mL})$ were added. The septum was removed and to the flask was added $\mathrm{MnO}_{2}(1.304 \mathrm{~g}, 15 \mathrm{mmol})$. The flask was then sealed with septum and DBU $(0.015 \mathrm{~mL}, 0.10 \mathrm{mmol})$ was added via syringe. The reaction stirred at ambient temperature until allylic alcohol and aldehyde were consumed (monitored by GC). The mixture was filtered through a thin pad of Celite. The Celite was washed with ethyl acetate $(15 \mathrm{ml})$. The filtrate was then concentrated in vacuo. The resulting residue was purified by flash chromatography on silica gel.

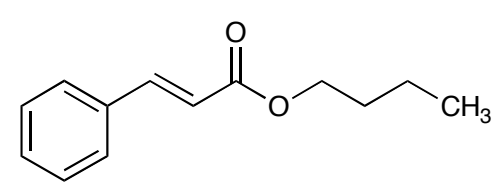

n-Butyl trans-cinnamate (6a): Filtrate was washed with $\mathrm{H}_{2} \mathrm{O}$ (2x), sat. $\mathrm{NaCl}$, dried with $\mathrm{Na}_{2} \mathrm{SO}_{4}$. Volatiles were removed yielding $190 \mathrm{mg}(93 \%)$ of $\mathbf{6 a}$ as a colorless oil. Spectral data matched those found in the literature. ${ }^{5}$

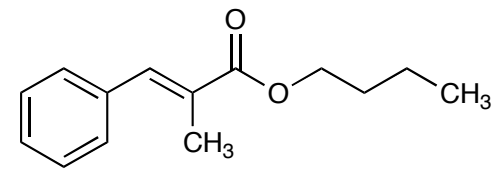

n-Butyl 2-methyl-3-phenyl-propenoate (6b): $25 \mathrm{~mol} \%$ triazolium salt used. Purified by column chromatography (8\% ethyl acetate/hexanes) yielding $199 \mathrm{mg}(91 \%)$ of $\mathbf{6 b}$ as a clear oil. $\mathrm{R}_{f}=0.65$ (17:3 hexanes/EtOAc); IR (film) 3175, 3038, 2963, 2873, 1710, 1636, $1449 \mathrm{~cm}^{-1} ;{ }^{1} \mathrm{H}$ NMR $\left(500 \mathrm{MHz}, \mathrm{CDCl}_{3}\right) \delta 7.69(\mathrm{~s}, 1 \mathrm{H}, \mathrm{HC}=\mathrm{C}) ; 7.40(\mathrm{~m}, 4 \mathrm{H})$; $7.32(\mathrm{~m}, 1 \mathrm{H}) ; 4.23\left(\mathrm{t}, 2 \mathrm{H}, \mathrm{OCH}_{2}\right) ; 2.12\left(\mathrm{~s}, 3 \mathrm{H}, \mathrm{C}=\mathrm{CCH}_{3}\right) ; 1.72\left(\mathrm{~m}, 2 \mathrm{H}, \mathrm{CH}_{2}\right) ; 1.46\left(\mathrm{~m}, 2 \mathrm{H}, \mathrm{CH}_{2}\right)$; $0.98\left(\mathrm{t}, 3 \mathrm{H}, \mathrm{CH}_{3}\right) ;{ }^{13} \mathrm{C} \mathrm{NMR}\left(500 \mathrm{MHz}, \mathrm{CDCl}_{3}\right) \delta$ 168.9, 138.8, 136.1, 129.8, 128.8, 128.5, 128.4, 65.0, 31.0, 19.5, 14.2; LRMS (electrospray): Mass calculated for $\mathrm{C}_{14} \mathrm{H}_{18} \mathrm{O}_{2}[3 \mathrm{M}+\mathrm{Na}]^{+} 677$. Found 676.8<smiles>CCCCOC(=O)c1cccc2ccccc12</smiles>

n-Butyl 1-naphthoate (7): Filtrate was washed with $\mathrm{H}_{2} \mathrm{O}(2 \mathrm{x})$, sat. $\mathrm{NaCl}$, dried with $\mathrm{Na}_{2} \mathrm{SO}_{4}$. Volatiles were removed yielding $204 \mathrm{mg}$

3. Kerr, M. S.; de Alaniz, J. R.; Rovis, T. J. Org. Chem. 2005, 70, 5725-5728.

4. Carlin, R. B.; Moores, M. S. J. Am. Chem. Soc. 1962, 84, 4107-4112.

5. Calo, V.; Nacci, A.; Monopoli, A.; Ieva, E.; Cioffi, N. Org. Lett. 2005, 7, 617-620. 
$(90 \%)$ of $\mathbf{7}$ as a white solid. Spectral data matched those found in the literature. ${ }^{6}$<smiles>CCCCOC(=O)c1ccc2ccccc2c1</smiles>

n-Butyl 2-naphthoate (8): Filtrate was washed with $\mathrm{H}_{2} \mathrm{O}(2 \mathrm{x})$, sat. $\mathrm{NaCl}$, dried with $\mathrm{Na}_{2} \mathrm{SO}_{4}$. Volatiles were removed yielding $207 \mathrm{mg}(91 \%)$ of $\mathbf{8}$ as a white solid. $\mathrm{R}_{f}=0.6 \quad(8: 2$ hexanes/EtOAc); IR (film) 3054, 2962, 1712, $1264 \mathrm{~cm}^{-1} ;{ }^{1} \mathrm{H}$ NMR

$\left(500 \mathrm{MHz}, \mathrm{CDCl}_{3}\right) \delta 8.21(\mathrm{~s}, 1 \mathrm{H}) ; 8.08(\mathrm{~m}, 1 \mathrm{H}) ; 7.87(\mathrm{~m}, 2 \mathrm{H}) ; 7.55(\mathrm{~m}, 2 \mathrm{H}) ; 4.40(\mathrm{t}, J=7,2 \mathrm{H})$; $1.82(\mathrm{~m}, 2 \mathrm{H}) ; 1.53(\mathrm{~m}, 2 \mathrm{H}) ; 1.02(\mathrm{t}, J=7,3 \mathrm{H}) ;{ }^{13} \mathrm{C} \mathrm{NMR}\left(500 \mathrm{MHz}, \mathrm{CDCl}_{3}\right) \delta$ 167.11, 135.73, 132.76, 131.21, 131.18, 129.60, 128.41, 128.35, 128.01, 126.85, 125.52, 65.26, 31.11, 19.60, 14.09; LRMS (electrospray): Mass calculated for $\mathrm{C}_{15} \mathrm{H}_{16} \mathrm{O}_{2}[2 \mathrm{M}]^{+} 456$. Found 456.2

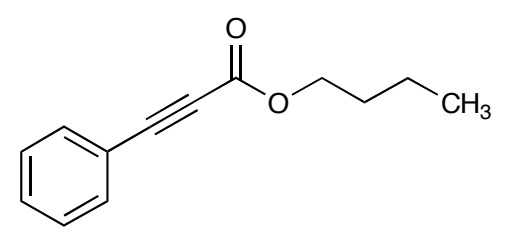

n-Butyl 3-phenylpropiolate (9): Purified by column chromatography (15\% ether/hexanes) yielding $171 \mathrm{mg}(85 \%)$ of 9 as a yellow oil. IR (film) 3054, 2963, 2221, 1706, $1273 \mathrm{~cm}^{-1}$; ${ }^{1} \mathrm{H} \mathrm{NMR}\left(500 \mathrm{MHz}, \mathrm{CDCl}_{3}\right) \delta$ 7.3-7.6 (m, 5H); $4.23(\mathrm{t}, 2 \mathrm{H}) ; 1.69$ $(\mathrm{m}, 2 \mathrm{H}) ; 1.42(\mathrm{~m}, 2 \mathrm{H}) ; 0.95(\mathrm{t}, 3 \mathrm{H}) ;{ }^{13} \mathrm{C} \mathrm{NMR}\left(500 \mathrm{MHz}, \mathrm{CDCl}_{3}\right)$ $\delta 154.2,132.9,130.8,128.5,119.6,86.0,80.6,65.9,30.4,19.0,14.1$; LRMS (electrospray): Mass calculated for $\mathrm{C}_{13} \mathrm{H}_{14} \mathrm{O}_{2}[2 \mathrm{M}+\mathrm{Na}]^{+} 427$. Found 427.8

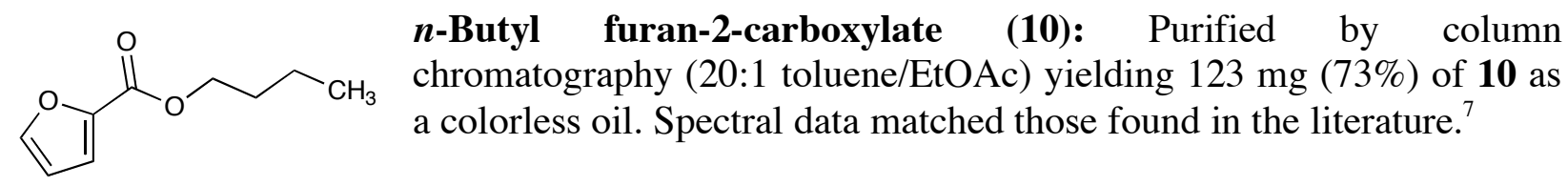

n-Butyl pent-2-enoate (11): Purified by column chromatography $\mathrm{H}_{3} \mathrm{C} \sim \mathrm{N}_{\mathrm{O}}^{\mathrm{O}} \sim \mathrm{CH}_{\mathrm{CH}_{3}}$ (15\% ether/hexanes) yielding $136 \mathrm{mg}(87 \%)$ of $\mathbf{1 1}$ as a colorless oil. IR (film) 3054, 2963, 2873, 1723, 1654, $1262 \mathrm{~cm}^{-1} ;{ }^{1} \mathrm{H}$ NMR $\left(500 \mathrm{MHz}, \mathrm{CDCl}_{3}\right) \delta 7.15\left(\mathrm{dt}, J=15.9,6.4,1 \mathrm{H}, \mathrm{CH}_{2} \mathrm{CH}\right) ; 5.81(\mathrm{~d}$, $J=15.9,1 \mathrm{H}, \mathrm{CH}) ; 4.13\left(\mathrm{t}, 2 \mathrm{H}, \mathrm{OCH}_{2}\right) ; 2.22\left(\mathrm{~m}, 2 \mathrm{H}, \mathrm{CH}_{2}\right) ; 1.64\left(\mathrm{~m}, 2 \mathrm{H}, \mathrm{CH}_{2}\right) ; 1.40\left(\mathrm{~m}, 2 \mathrm{H}, \mathrm{CH}_{2}\right)$; $1.07\left(\mathrm{t}, 3 \mathrm{H}, \mathrm{CH}_{3}\right) ; 0.94\left(\mathrm{t}, 3 \mathrm{H}, \mathrm{CH}_{3}\right) ;{ }^{13} \mathrm{C} \mathrm{NMR}\left(500 \mathrm{MHz}, \mathrm{CDCl}_{3}\right) \delta 167.0,150.6,120.3,64.1$, 30.7, 25.3, 19.2, 13.7, 12.1; LRMS (electrospray): Mass calculated for $\mathrm{C}_{9} \mathrm{H}_{16} \mathrm{O}_{2}[2 \mathrm{M}+\mathrm{Na}]^{+} 335$. Found 335.6

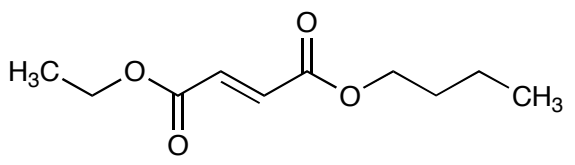

n-Butyl ethyl fumarate (12): Purified by column chromatography (10\% ether/hexanes) yielding $130 \mathrm{mg}$ $(65 \%)$ of $\mathbf{1 2}$ as colorless oil. Spectral data matched those found in the literature. ${ }^{8}$

6. Cai, M. Z.; Song, C. S.; Huang, X. J. Chem. Soc. Perkin Trans. 1 1997, 2273-2274.

7. Pittelkow, M.; Kamounah, F. S.; Boas, U.; Pedersen, B.; Christensen, J. B. Synthesis 2004, 2485-2492.

8. Zaderenko, P.; Lopez, M. C.; Ballesteros, P. J. Org. Chem. 1996, 61, 6825-6828. 
<smiles>CCCCOC(=O)c1ccccc1</smiles>

literature. ${ }^{9}$<smiles>CCCCOC(=O)c1ccccc1Br</smiles>

n-Butyl benzoate (13): The standard procedure was followed with 2 mmol benzyl alcohol and $50 \mathrm{~mol} \%$ triazolium and DBU. Purified by column chromatography (20:1 toluene/EtOAc) to yield $313 \mathrm{mg}(88 \%)$ of $\mathbf{1 3}$ as colorless oil. Spectral data matched those found in the

n-Butyl 2-bromobenzoate (14): $25 \mathrm{~mol} \%$ of triazolium salt used. Purified by column chromatography (15\% ether/hexanes) yielding 180 $\mathrm{mg}(70 \%)$ of 14 as a yellow oil. IR (film) 3054, 2962, 1729, 1696, 1271, $705 \mathrm{~cm}^{-1}$; ${ }^{1} \mathrm{H}$ NMR $\left(500 \mathrm{MHz}, \mathrm{CDCl}_{3}\right) \delta$ 7.3-7.9 (m, 4H); $4.34(\mathrm{t}$, $\left.2 \mathrm{H}, \mathrm{OCH}_{2}\right) ; 1.75\left(\mathrm{~m}, 2 \mathrm{H}, \mathrm{CH}_{2}\right) ; 1.48\left(\mathrm{~m}, 2 \mathrm{H}, \mathrm{CH}_{2}\right) ; 0.97\left(\mathrm{t}, 3 \mathrm{H}, \mathrm{CH}_{3}\right)$; ${ }^{13} \mathrm{C}$ NMR $\left(500 \mathrm{MHz}, \mathrm{CDCl}_{3}\right.$ ) $\delta 166.4,135.3,134.3,132.4,127.9,127.1,121.6,65.5,30.6,19.3$, 13.7; LRMS (electrospray): Mass calculated for $\mathrm{C}_{11} \mathrm{H}_{13} \mathrm{O}_{2} \mathrm{Br}[2 \mathrm{M}+\mathrm{Na}]^{+} 537.2$ Found 537.7

\section{General Procedure for triazolium-catalyzed oxidation with toluene as solvent:}

A flame-dried round bottom flask was charged with triazolium salt $(34 \mathrm{mg}, 0.15 \mathrm{mmol})$. The flask was sealed with a septum and put under positive pressure of nitrogen. Toluene $(5 \mathrm{ml})$, the allylic alcohol $(1 \mathrm{mmol})$, and the nucleophilic alcohol $(5 \mathrm{mmol})$ were added. The septum was removed and to the flask was added $\mathrm{MnO}_{2}(1.304 \mathrm{~g}, 15 \mathrm{mmol})$. The flask was then sealed with septum and DBU $(0.165 \mathrm{ml}, 1.1 \mathrm{mmol})$ was added via syringe. The reaction stirred at ambient temperature until allylic alcohol and aldehyde were consumed (monitored by GC). Upon consumption of allylic alcohol and aldehyde, methanol $(10 \mathrm{~mL})$ was added to the reaction. The mixture was then quickly filtered through a thin pad of celite (prolonged stirring with methanol resulted in transesterification to the methyl ester). The Celite was washed with ethyl acetate (15 $\mathrm{ml})$. The filtrate was then concentrated in vacuo. The resulting residue was purified by flash chromatography on silica gel.

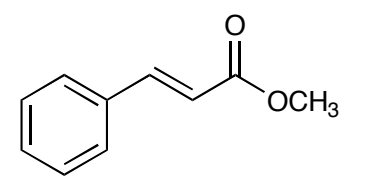

Methyl trans-cinnamate (5): Filtration through Celite and concentration in vacuo yielded $154 \mathrm{mg}(95 \%)$ of $\mathbf{5}$ as a white solid. Spectral data matched those found in the literature. ${ }^{10}$<smiles>CC(C)OC(=O)/C=C/c1ccccc1</smiles>

2-Propyl trans-cinnamate (15): Cinnamyl alcohol combined with $\mathrm{MnO}_{2}$ in toluene and stirred for 30 minutes (until complete consumption of alcohol) at ambient temperature. Triazolium salt, DBU, and 2-propanol were added and stirred. Reaction mixture stirred with methanol and quickly filtered through Celite. Filtrate was concentrated in vacuo yielding $169 \mathrm{mg}(89 \%)$ of $\mathbf{1 5}$ as a yellow oil. Spectral data matched those found in the literature. ${ }^{11}$

9. Hans, J. J.; Driver, R. W.; Burke, S. D. J. Org. Chem. 2000, 65, 2114-2121.

10. Crosignani, S.; White, P. D.; Linclau, B. Org. Lett. 2002, 4, 2961-2963. 
<smiles>O=C(/C=C/c1ccccc1)OCC(Cl)(Cl)Cl</smiles>

2,2,2-Trichloroethyl trans-cinnamate (16): Purified by column chromatography (20:1 toluene/EtOAc) yielding $228 \mathrm{mg}(82 \%)$ of $\mathbf{1 6}$ as colorless crystals. Spectral data matched those found in the literature. ${ }^{12}$<smiles>COCCOC(=O)/C=C/c1ccccc1</smiles>

2-Methoxyethyl trans-cinnamate (17): Purified by column chromatography (20:1 toluene/EtOAc) yielding $169 \mathrm{mg}(82 \%)$ of 17 as a yellow oil. $\mathrm{R}_{f}=0.33$ (8:2 hexanes/EtOAc); IR (film) $3054,2986,2932,1710,1633,1300,1250,1200,1180 \mathrm{~cm}^{-1} ;{ }^{1} \mathrm{H}$ NMR $\left(500 \mathrm{MHz}, \mathrm{CDCl}_{3}\right) \delta 7.72(\mathrm{~d}, J=16,1 \mathrm{H}) ; 7.52(\mathrm{~m}, 2 \mathrm{H})$; $7.38(\mathrm{~m}, 3 \mathrm{H}) ; 6.50(\mathrm{~d}, J=16,1 \mathrm{H}) ; 4.37(\mathrm{t}, J=4,2 \mathrm{H}) ; 3.67(\mathrm{t}, J=4,2 \mathrm{H}) ; 3.43(\mathrm{~s}, 3 \mathrm{H}) ;{ }^{13} \mathrm{C}$ NMR $\left(500 \mathrm{MHz}, \mathrm{CDCl}_{3}\right) \delta 167.24,145.40,134.60,130.59,129.15,128.37,118.03,70.82,63.84$, 59.32; LRMS (electrospray): Mass calculated for $\mathrm{C}_{12} \mathrm{H}_{14} \mathrm{O}_{3}[2 \mathrm{M}+\mathrm{Na}]^{+} 435$. Found 435.2

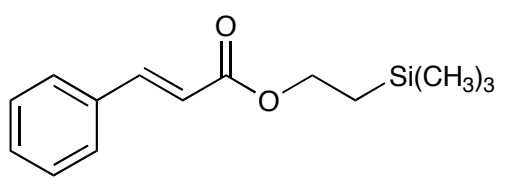

2-(Trimethylsilyl)ethyl trans-cinnamate (18): Purified by column chromatography (15\% ether/hexanes) yielding $183 \mathrm{mg}$ (74\%) of 18 as a yellow oil. IR (film) 3054, 2956, 1706, 1638, $1268 \mathrm{~cm}^{-1} ;{ }^{1} \mathrm{H}$ NMR $\left(500 \mathrm{MHz}, \mathrm{CDCl}_{3}\right) \delta 7.68(\mathrm{~d}, J=15.9,1 \mathrm{H}$, $\mathrm{CH}) ; 7.3-7.5(\mathrm{~m}, 5 \mathrm{H}) ; 6.43(\mathrm{~d}, J=15.9,1 \mathrm{H}, \mathrm{CH}) ; 4.31(\mathrm{t}, J=8.5$, $\left.2 \mathrm{H}, \mathrm{OCH}_{2}\right) ; 1.08\left(\mathrm{t}, J=8.5,2 \mathrm{H}, \mathrm{CH}_{2} \mathrm{Si}\right) ; 0.08\left(\mathrm{~s}, 9 \mathrm{H}, \mathrm{Si}\left(\mathrm{CH}_{3}\right)_{3}\right) ;{ }^{13} \mathrm{C} \mathrm{NMR}\left(500 \mathrm{MHz}, \mathrm{CDCl}_{3}\right) \delta$ $167.0,144.4,134.4,130.1,128.8,128.0,118.4,62.6,17.3,-1.4,-1.6,-1.9$; LRMS (electrospray): Mass calculated for $\mathrm{C}_{14} \mathrm{H}_{20} \mathrm{O}_{2} \mathrm{Si}[2 \mathrm{M}+\mathrm{Na}]^{+} 519.8$ Found 519.4<smiles>COC(=O)CCc1ccccc1</smiles>

Methyl 3-phenylpropionate (19): A flame-dried round bottom flask was charged with triazolium salt $(22 \mathrm{mg}, 0.10 \mathrm{mmol})$. The flask was sealed with a septum and put under positive pressure of nitrogen. Hydrocinnamaldehyde $(0.5 \mathrm{mmol})$, and methanol $(2.5 \mathrm{~mL})$ were added. The septum was removed and to the flask was added $\mathrm{MnO}_{2}(652 \mathrm{mg}, 7.5$ mmol). The flask was then sealed with septum and DBU $(0.015 \mathrm{~mL}, 0.10 \mathrm{mmol})$ was added via syringe. The reaction stirred at ambient temperature until aldehyde was consumed (monitored by TLC). The mixture was filtered through a thin pad of Celite. The Celite was washed with ethyl acetate $(15 \mathrm{ml})$. The filtrate was then concentrated in vacuo to afford $75 \mathrm{mg}(91 \%) 19$ as a yellow oil. Spectral data matched those found in the literature. ${ }^{13}$

\section{General Procedure for NHC-catalyzed desymmetrization of meso diols:}

11. Shono, T.; Ishige, O.; Uyama, H.; Kashimura, S. J. Org. Chem. 1986, 51, 546-549.

12. Dambacher, J.; Zhao, W.; El-Batta, A.; Anness, R.; Jiang, C. C.; Bergdahl, M. Tetrahedron Lett. 2005, 46, 4473-4477.

13. Semmelhack, M. F.; Stauffer, R. D.; Yamashita, A. J. Org. Chem. 1977, 42, 3180-3188. 
A flame-dried $10 \mathrm{ml}$ round bottom flask equipped with stirbar was charged with the chiral triazolium salt $\mathbf{E}$ (50 $\mathrm{mg}, 0.119 \mathrm{mmol}), \mathrm{K}_{2} \mathrm{CO}_{3}(16.4 \mathrm{mg}, 0.119 \mathrm{mmol})$, and 18-crown-6 (15.7 $\mathrm{mg}, 0.06 \mathrm{mmol})$. The flask was sealed with a septum and placed under a positive pressure of nitrogen. Into it was then added the meso diol $(115 \mathrm{mg}, 0.99 \mathrm{mmol})$, proton sponge $(85 \mathrm{mg}$, $0.397 \mathrm{mmol}$ ), and $\mathrm{MnO}_{2}(517 \mathrm{mg}, 5.95 \mathrm{mmol})$. Dichloromethane $(1.6 \mathrm{~mL}, 0.25 \mathrm{M})$ was added resulting in a black suspension. Lastly, cinnamaldehyde $(50 \mu \mathrm{L}, 0.397 \mathrm{mmol})$ was added via syringe. The reaction was allowed to stir at $-30{ }^{\circ} \mathrm{C}$ under a positive pressure of nitrogen until the aldehyde was consumed as determined by TLC. Upon consumption of the aldehyde, the mixture was filtered through a thin pad of celite and washed with ethyl acetate $(25 \mathrm{~mL})$. The filtrate was then concentrated in vacuo and the resulting residue was purified by flash chromatography on silica gel.

(E)-(1S,2R)-2-hydroxycyclohexyl cinnamate (21): Purified by column chromatography (25\% EtOAc/hexane) yielding $57 \mathrm{mg}$ (58\%) of 21 as a white solid. Mp: $61-63{ }^{\circ} \mathrm{C}$; IR (film) 3464 , 3048, 2936, 2861, 2351, 1701, $1281 \mathrm{~cm}^{-1}$; ${ }^{1} \mathrm{H}$ NMR (400 MHz, $\mathrm{CDCl}_{3}$ ) $\delta 7.10$ (d, J=15.8, $1 \mathrm{H}$, $\mathrm{CH}) ; 7.53(\mathrm{~m}, 2 \mathrm{H}) ; 7.39(\mathrm{~m}, 3 \mathrm{H}) ; 6.58(\mathrm{~d}, J=16.4,1 \mathrm{H}, \mathrm{CH}) ; 5.07(\mathrm{~m}, 1 \mathrm{H}) ; 3.95(\mathrm{~m}, 1 \mathrm{H}) ; 1.98-$ $1.95(\mathrm{~m}, 2 \mathrm{H}) ; 1.84-1.82(\mathrm{~m}, 1 \mathrm{H}) ; 1.69-1.65(\mathrm{~m}, 3 \mathrm{H}) ; 1.57(\mathrm{~s}, 1 \mathrm{H}) ; 1.43-1.39(\mathrm{~m}, 2 \mathrm{H}) ;{ }^{13} \mathrm{C}$ NMR $\left(100 \mathrm{MHz}, \mathrm{CDCl}_{3}\right) \delta$ 166.9, 145.4, 134.5, 130.6, 129.1, 128.3, 118.3, 74.5, 69.5, 30.6, 27.3, 22.3, 21.3; LRMS (electrospray): Mass calculated for $\mathrm{C}_{15} \mathrm{H}_{18} \mathrm{O}_{3}[2 \mathrm{M}+\mathrm{Na}]^{+} 515.6$ Found 515.4

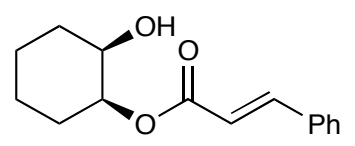

\section{Determination of Enantiomeric excess:}

The desymmetrization of cis-1,2-cyclohexanediol was carried out using cinnamaldehyde and the chiral catalyst $\mathbf{2 1}$ to yield $\mathbf{2 0}$ with an enantiomeric excess of $80 \%$. Enantiomeric excess determined by HPLC on a Chiralcel AD-H column. $10 \% \mathrm{IPA} / \mathrm{Hexanes}, 1 \mathrm{~mL} / \mathrm{min}$. 


\section{Racemic product:}
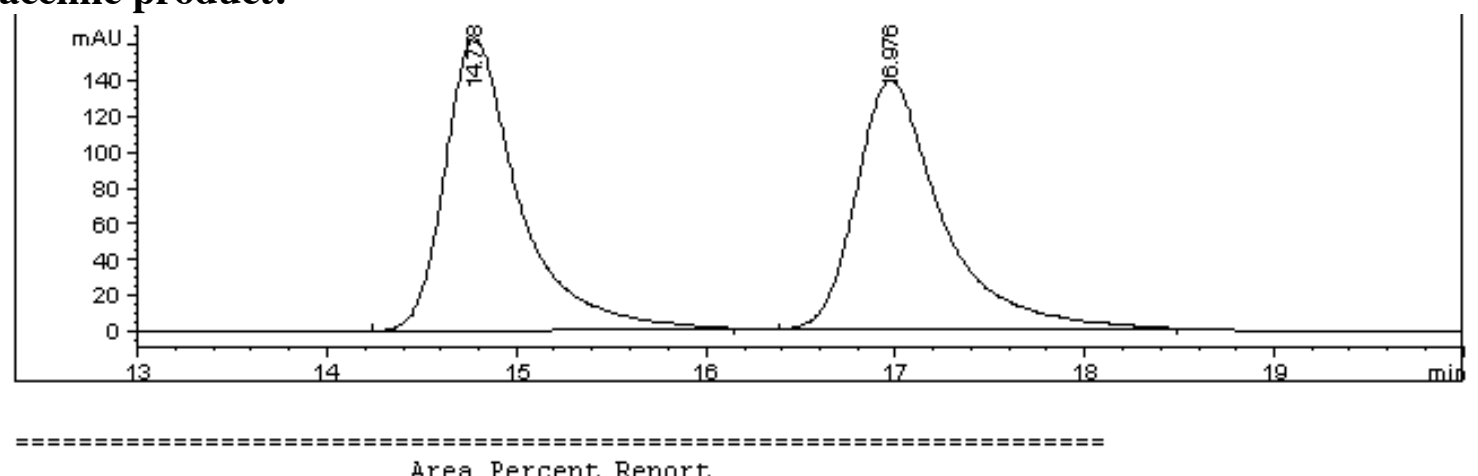

更

$\begin{array}{lll}\text { Sorted By } & : & \text { Sicnal } \\ \text { Multiplier } & : & 1.0000\end{array}$

1.0000

Dilution : 1.0000

Use Multiplier \& Dilution Factor with ISTDs

Signal 1: DẢDl Aे, Sig $=254,4$ Ref $=360,100$

\begin{tabular}{|c|c|c|c|c|c|c|}
\hline $\begin{array}{c}\text { Peak } \\
\#\end{array}$ & $\begin{array}{c}\text { RetTime } \\
\text { 「min } 1\end{array}$ & Type & $\begin{array}{l}\text { Width } \\
\text { 「min } 1\end{array}$ & $\begin{array}{c}\text { Area } \\
\left\lceil\mathrm{m} \dot{\mathrm{A} U} \mathrm{U}^{*} \mathrm{~S}\right\rceil\end{array}$ & $\begin{array}{l}\text { Height } \\
\text { 「mAU1 }\end{array}$ & Area \\
\hline " & ------- & & ------ & -------- & --ー-ー--ー- & $--------\mid$ \\
\hline 1 & 14.778 & $\mathrm{BB}$ & 0.4061 & 4483.20117 & 163.41731 & 50.5538 \\
\hline 2 & 16.976 & PB & 0.4681 & 4384.97656 & 138.98174 & 49.4462 \\
\hline Total & : & & & 8868.17773 & 302.39905 & \\
\hline
\end{tabular}

\section{Optically enriched product:}
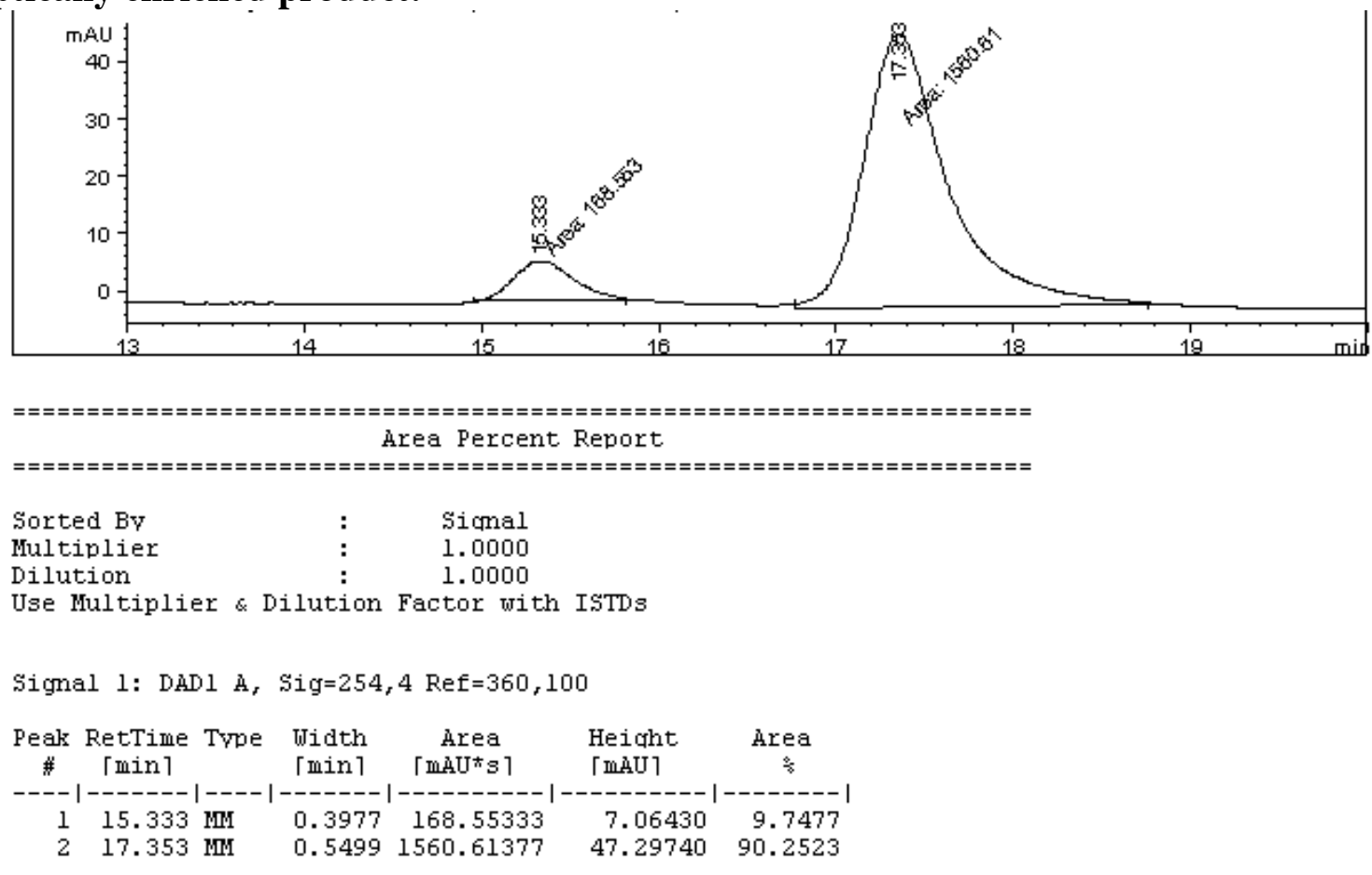

Totals :

$1729.16710 \quad 54.36171$ 


\section{Selected NMR Spectra:}

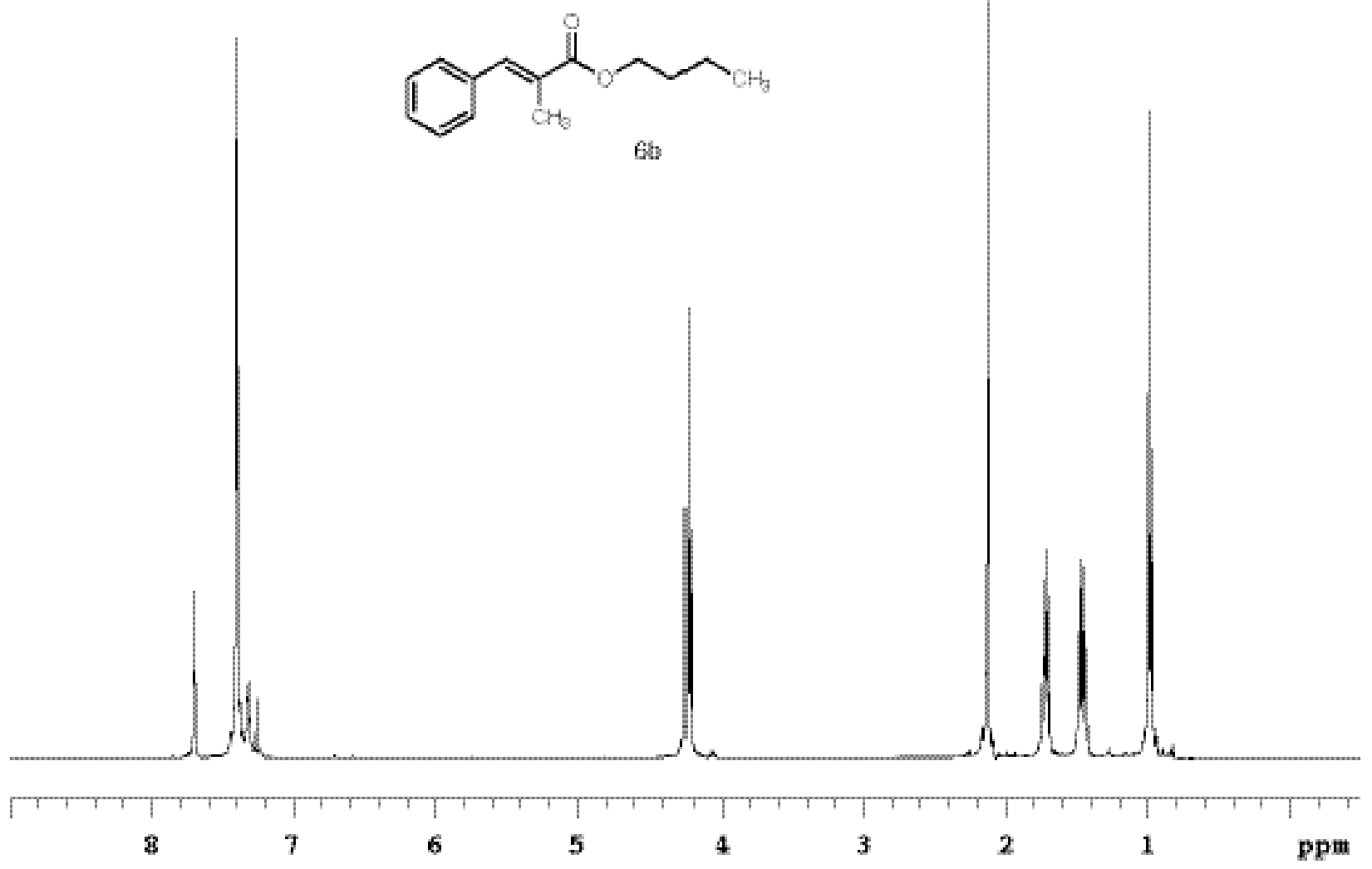




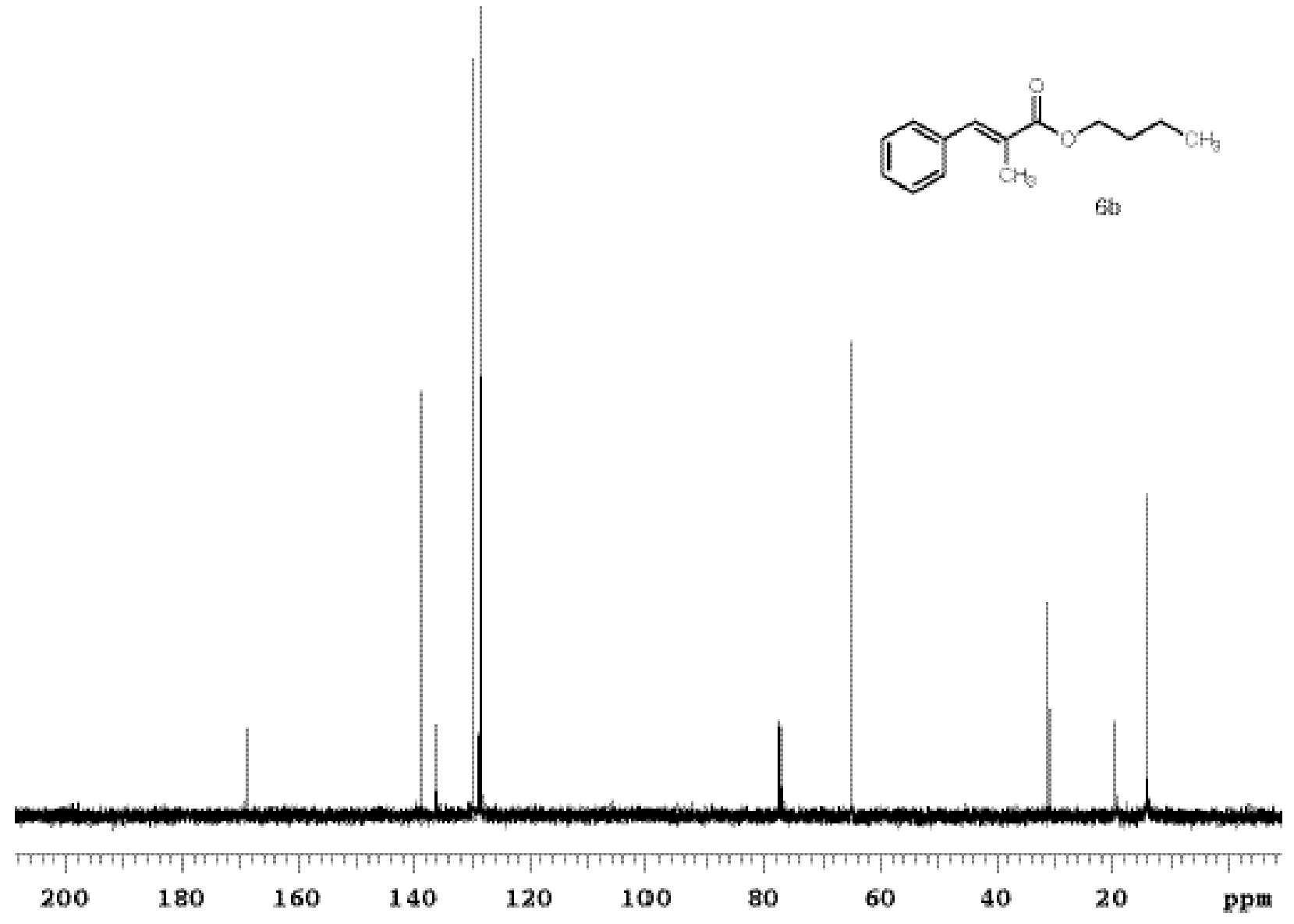




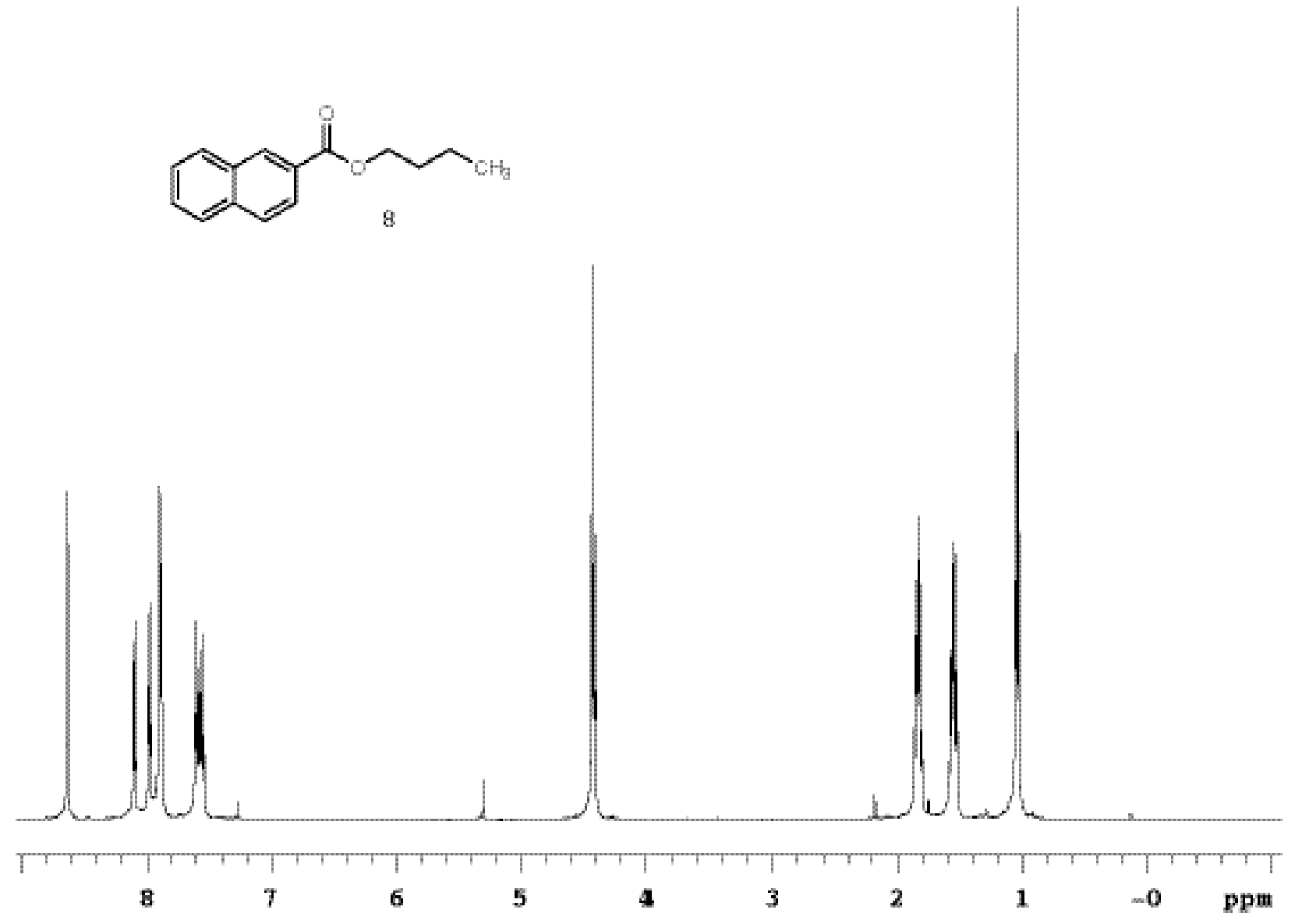




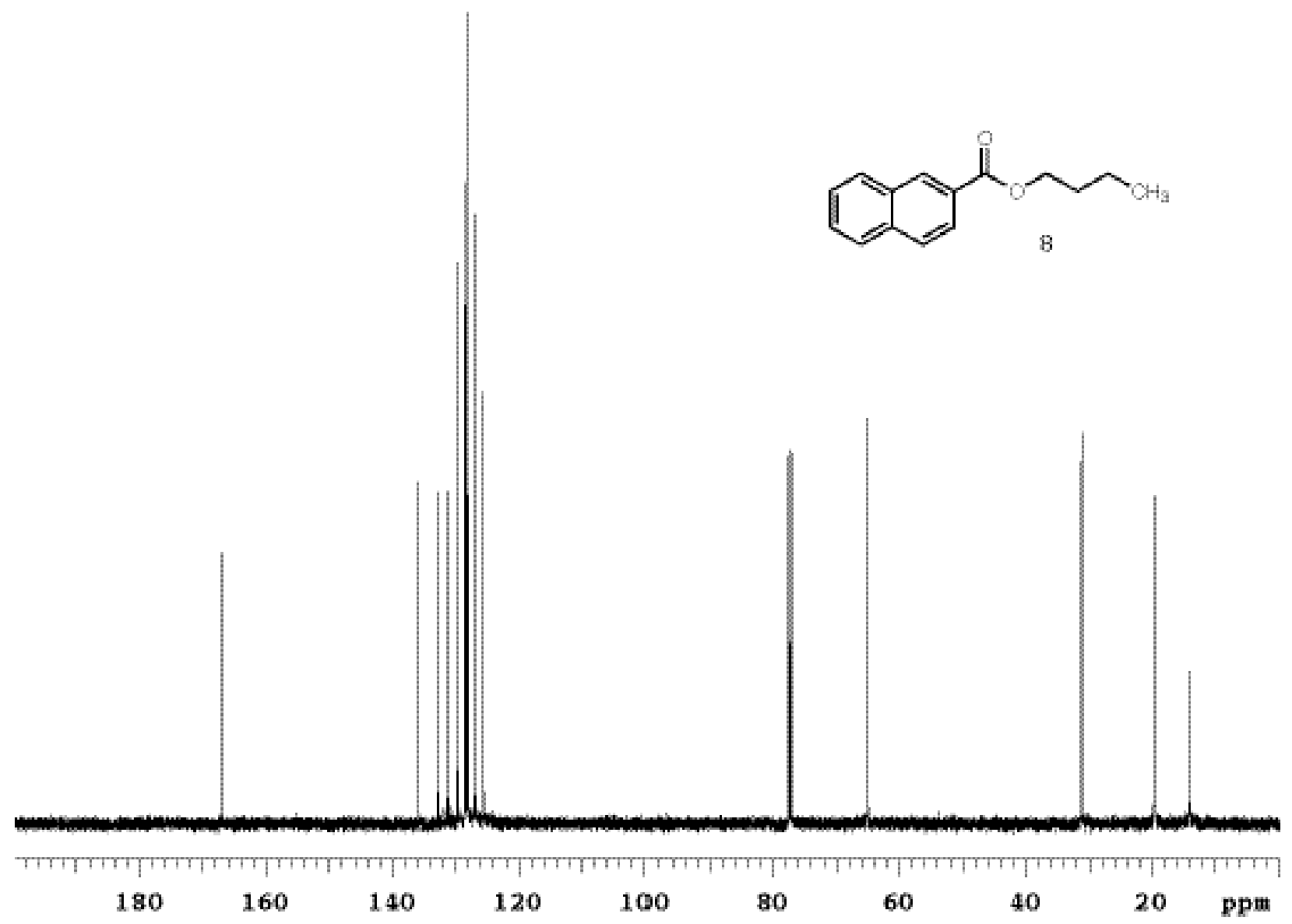




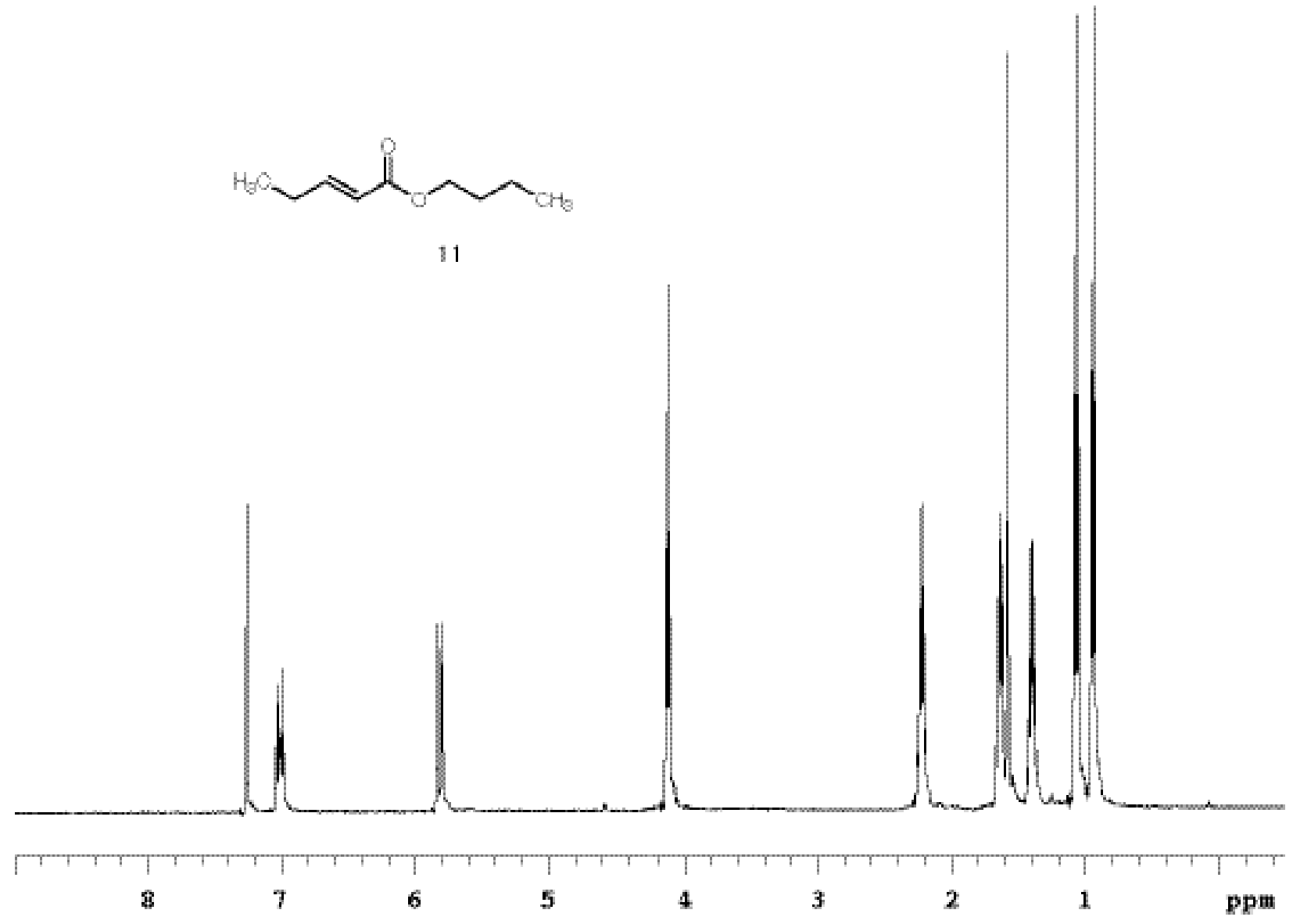




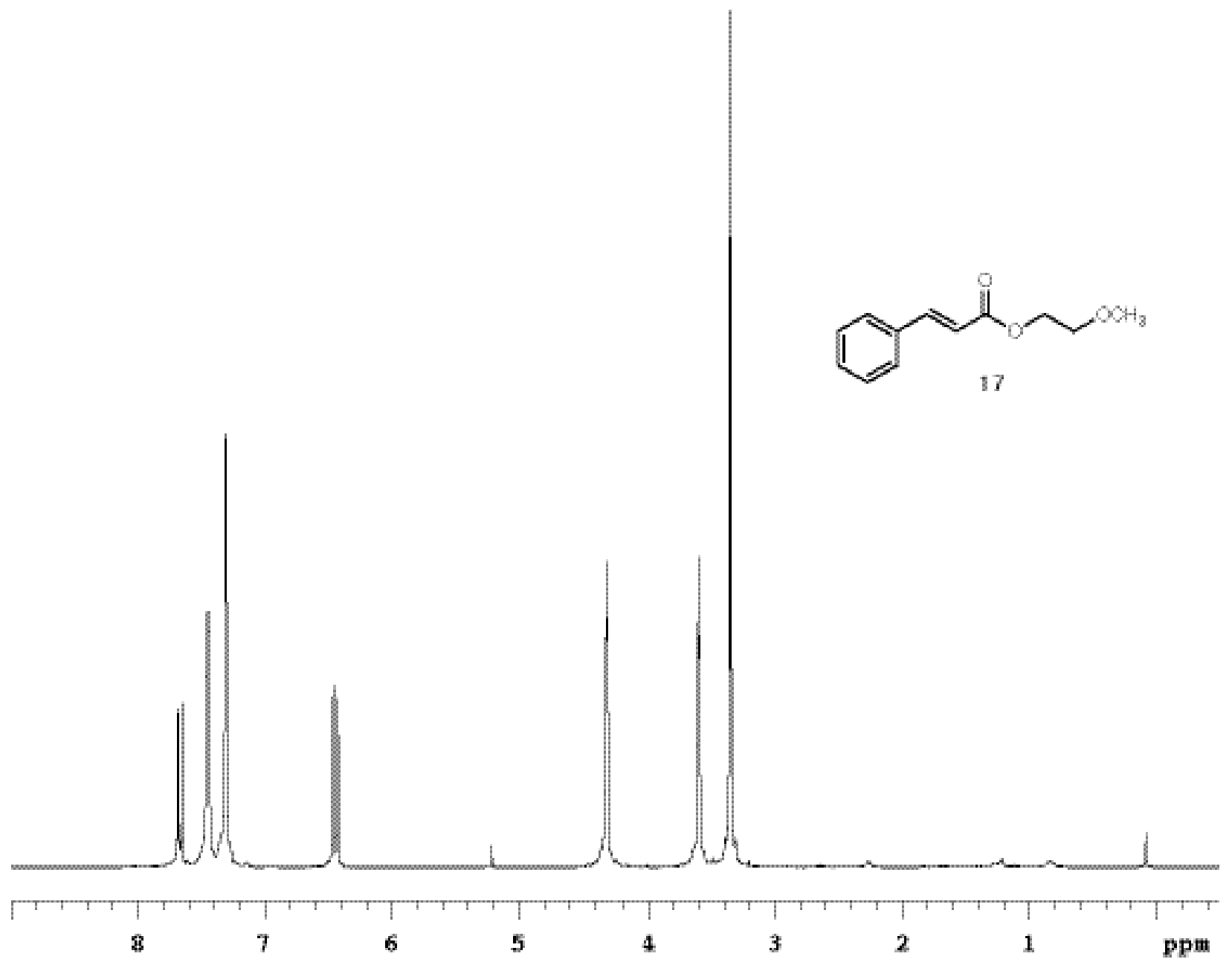




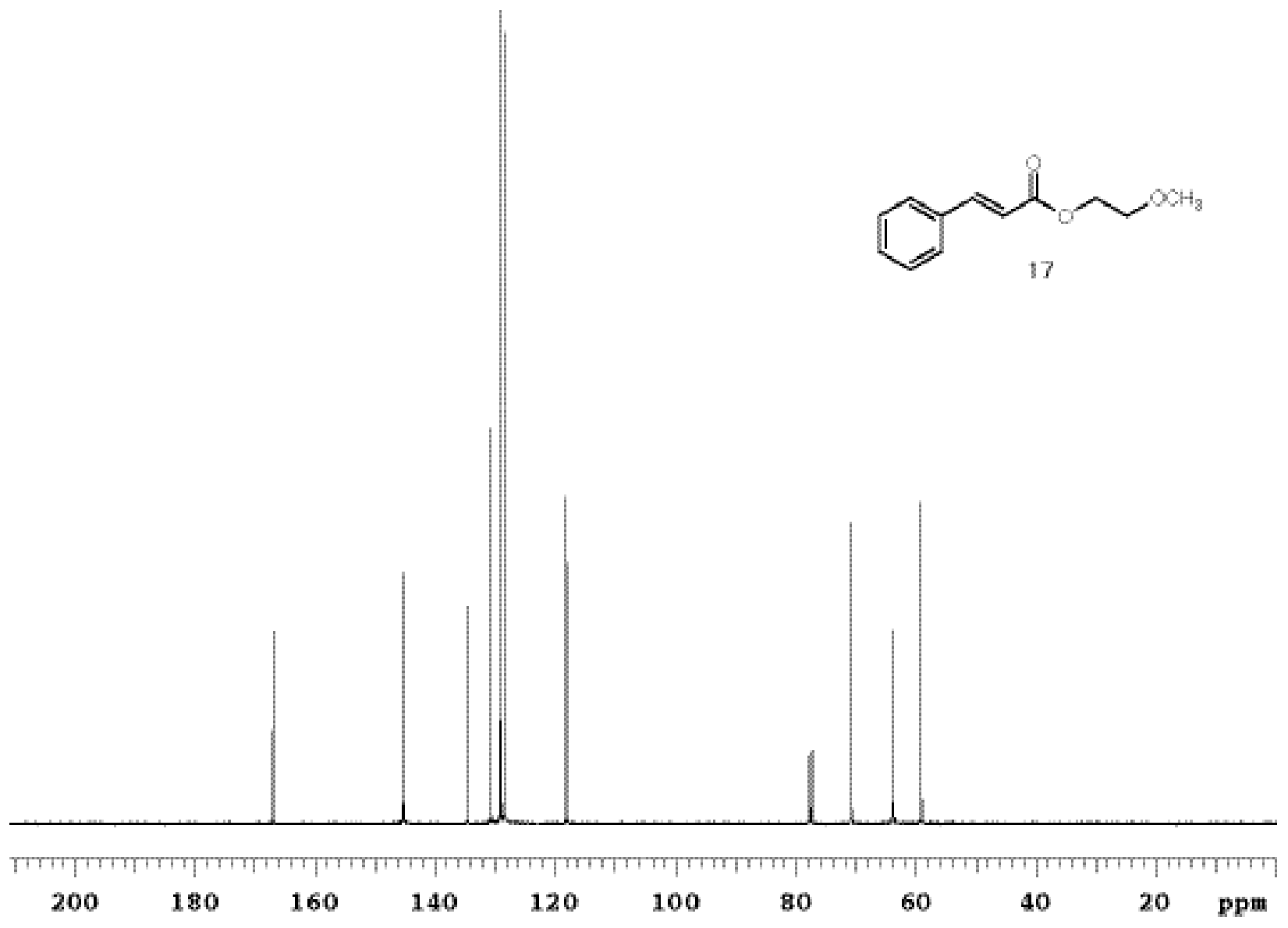




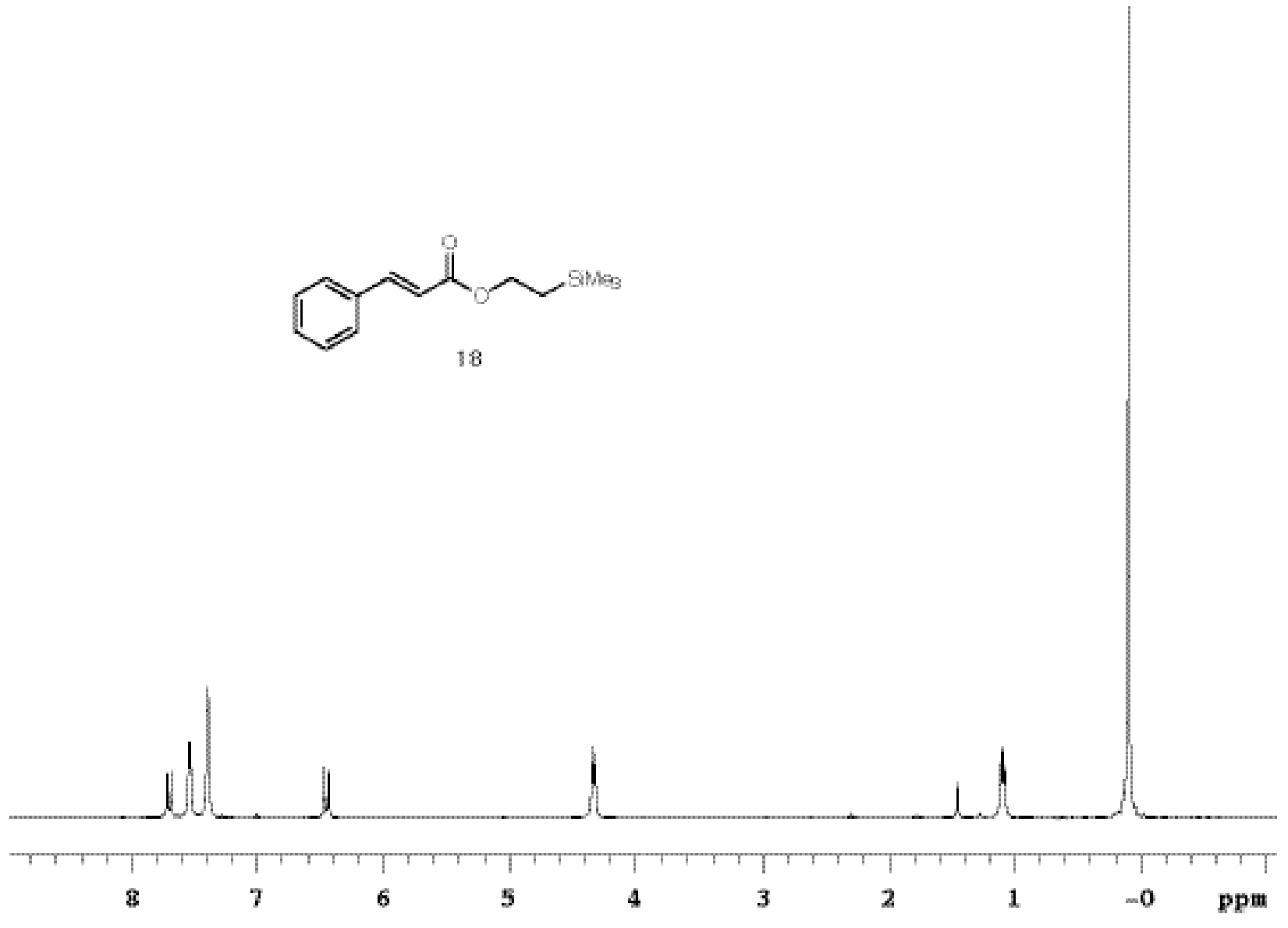




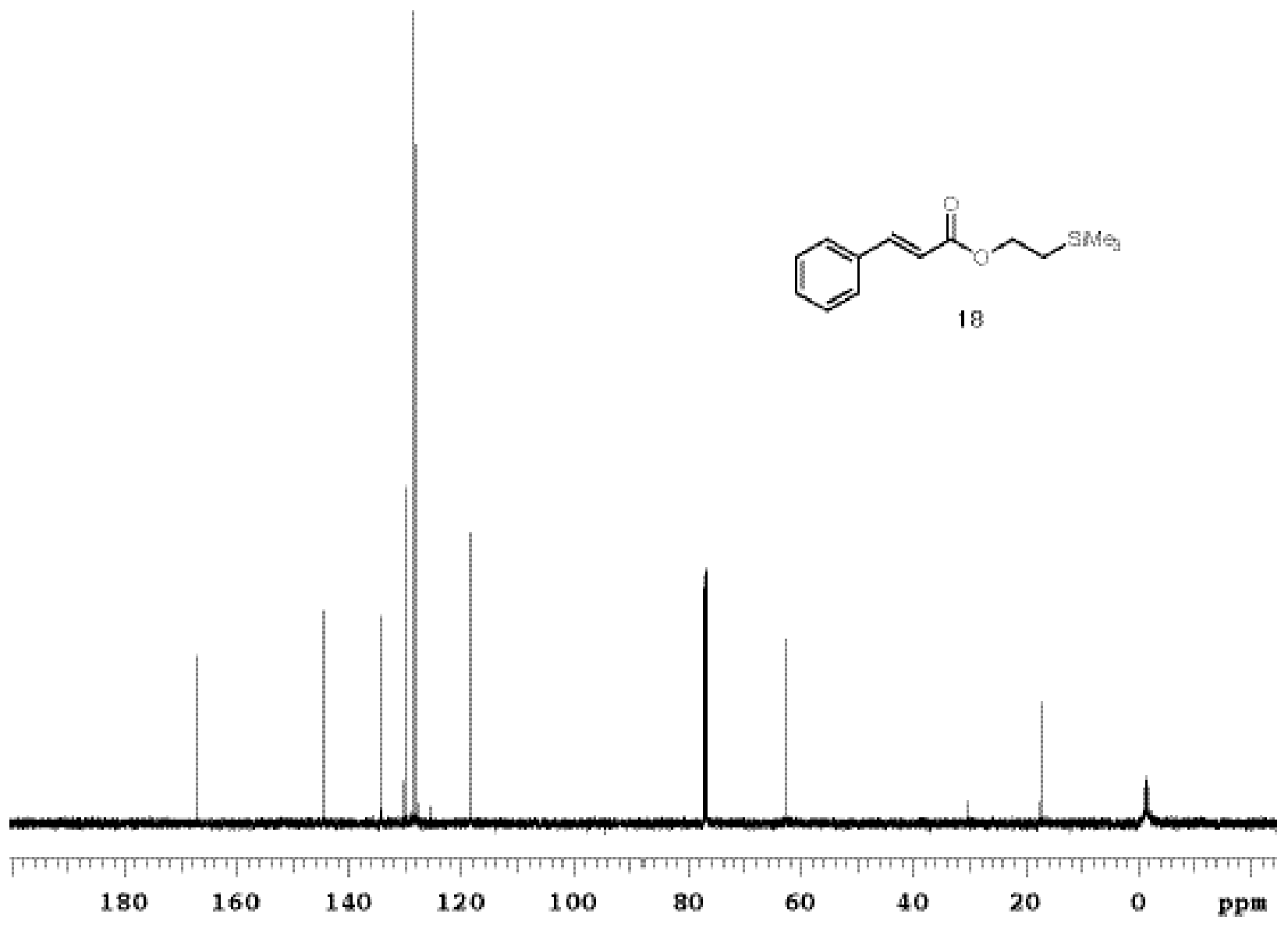




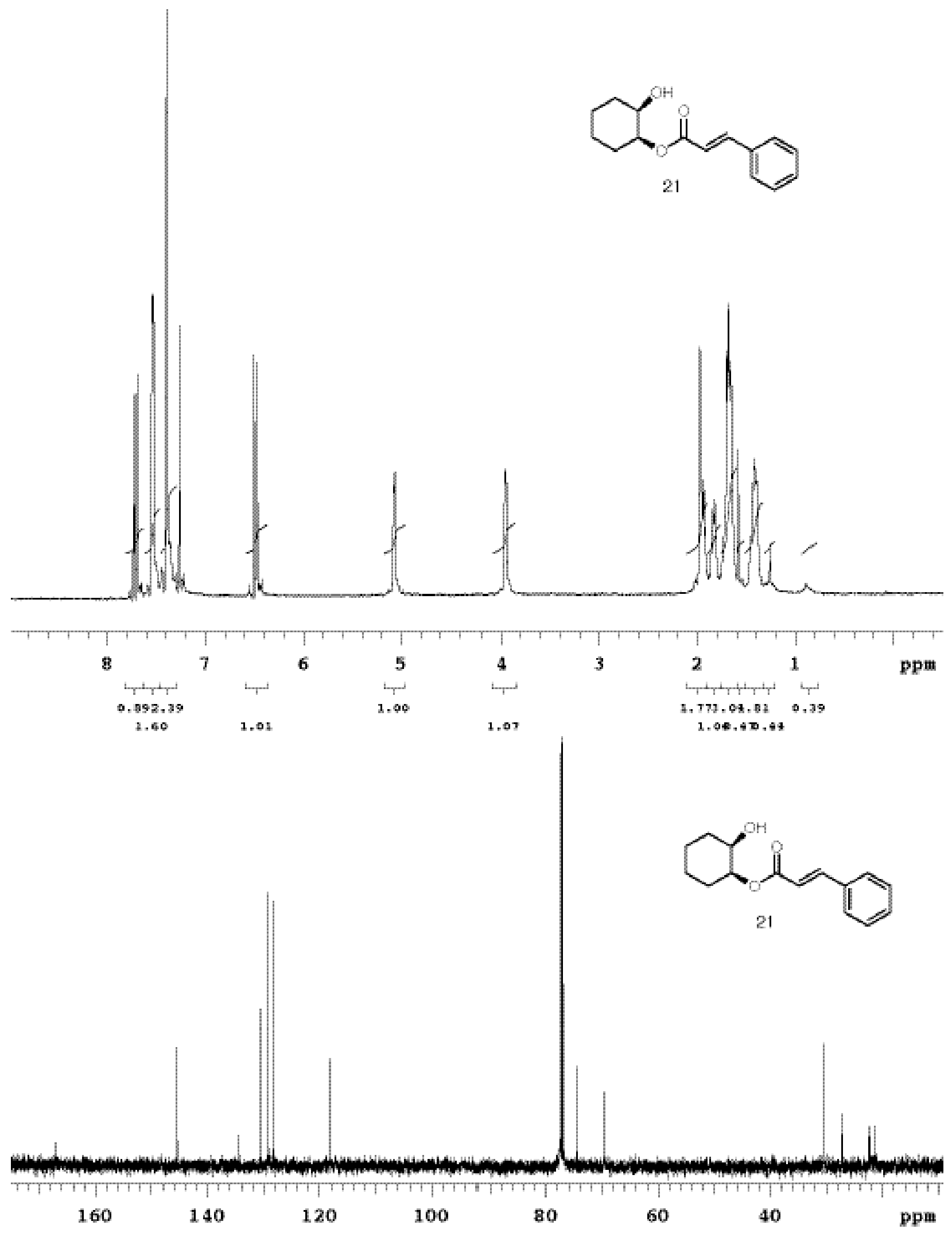

